\title{
Patrick HUCHET, Entre Moyen Âge et Renaissance, les grandes heures de Josselin
}

\section{Patrick Kernévez}

\section{CpenEdition}

\section{Journals}

Édition électronique

URL : https://journals.openedition.org/ccm/5426

DOI : $10.4000 / \mathrm{ccm} .5426$

ISSN : 2119-1026

\section{Éditeur}

Centre d'études supérieures de civilisation médiévale/Université de Poitiers

\section{Édition imprimée}

Date de publication : 1 décembre 2020

Pagination : 306-307

ISBN : 978-2-490783-07-6

ISSN : 0007-9731

\section{Référence électronique}

Patrick Kernévez, "Patrick нuснет, Entre Moyen Âge et Renaissance, les grandes heures de Josselin », Cahiers de civilisation médiévale [En ligne], 252 | 2020, mis en ligne le 01 décembre 2020, consulté le 10 décembre 2022. URL : http://journals.openedition.org/ccm/5426; DOI : https://doi.org/10.4000/ccm. 5426

\section{(c) (i) (9)}

Creative Commons - Attribution - Pas d'Utilisation Commerciale - Pas de Modification 4.0 International - CC BY-NC-ND 4.0

https://creativecommons.org/licenses/by-nc-nd/4.0/ 
Patrick HuchEt, Entre Moyen Âge et Renaissance, les grandes heures de Josselin, Rennes, Éditions OuestFrance, 2019.

L'ouvrage est un beau livre consacré à Josselin, petite cité de caractère de Bretagne centrale, successivement détenue par les vicomtes de Porhoët puis les vicomtes et ducs de Rohan, toujours propriétaires du château. C'est une de ces villes " nées à l'ombre d'un château » comme on en recense plusieurs dizaines dans cette province, avec des récits de fondation parfois enjolivés, tant leurs origines peuvent rester méconnues. L'a. retrace l'histoire du lieu et de ses monuments à travers cinq chapitres qui nous mènent du début $d u X^{e}{ }^{\mathrm{e}}$ s. à la fin du XVI ${ }^{\mathrm{e}}$ s., selon un plan chronologique également scandé par la succession des lignages et des personnages importants, comme Olivier de Clisson, connétable de France, propriétaire de la seigneurie de 1370 à 1407, ou Jean II de Rohan (1462-1516).

Pour nous offrir une synthèse agréable à lire et documentée, l'a. se fonde sur un certain nombre de travaux dont il compile des passages significatifs comme les volumes anciens d'Hervé du Halgouët consacrées aux Rohan (Hervé du HalgouËt, La Vicomté de Rohan et ses seigneurs : accompagnée d'une carte et de plans, Saint-Brieuc/Paris, René Prud'homme éditeur/Édouard Champion éditeur [Contribution à l'étude du régime seigneurial dans l'ancienne France], 1921; ID., Le Duché de Rohan et ses seigneurs, accompagné d'une carte et de notices sur les principales seigneuries du fief, Saint-Brieuc/ Paris, René Prud'homme éditeur/Édouard Champion éditeur [Contribution à l'étude du régime seigneurial dans l'ancienne France], 1924), la biographie aujourd'hui traduite que John Bell Henneman a consacrée au connétable de France (John B. HenNemAn, Olivier de Clisson et la société politique française sous les règnes de Charles V et Charles VI, P. Galliou [trad.] et M. Jones [préf.], Rennes, Presses universitaires de Rennes [Histoire], 2011), les ouvrages d'Yvonig Gicquel relatifs au même personnage et à deux autres propriétaires du château, Alain IX et Jean II de Rohan (Yvonig GicQuel, Olivier de Clisson : 1336-1407, connétable de France ou chef de parti breton?, Paris, J. Picollec, 1981 ; ID., Alain IX de Rohan [1382-1462], un grand seigneur de l'âge d'or de Bretagne, Paris, J. Picollec [Bibliothèque celtique], 1986; ID., Jean II de Rohan ou l'indépendance brisée de la Bretagne, J. de Rohan [préf.], Paris, J. Picollec, 1994), ou encore un travail non publié d'Arnaud Bourriquen consacré aux premiers seigneurs du château (Arnaud Bourriquen, Les vicomtes de Rennes et leurs descendants, les Porhoët/Rohan face au pouvoir ducal [fin $\left.x^{\mathrm{e}}-\mathrm{FIN} x i i^{\mathrm{e}}\right]$, mémoire de master 2, Université de Rennes 2, 2008) et d'autres encore. Ces emprunts lui permettent de brosser un tableau qui reste incomplet, en raison de la carence des sources inhérente à l'histoire de la Bretagne intérieure au Moyen Âge. Josselin semble ainsi surgir du néant au début du XI ${ }^{\mathrm{e}} \mathrm{s}$, à l'image de nombreux châteaux alors que l'on sait depuis les travaux d'Hubert Guillotel (Hubert Guillotel, « De la vicomté de Rennes à la vicomté de Porhoët [fin du X ${ }^{\mathrm{e}}$-milieu du XII ${ }^{\mathrm{e}}$ siècle] », Mémoires de la Société d'histoire et d'archéologie de Bretagne, LXXII, 1995, p. 5-23) que les origines de la ville ont été réécrites par les moines de Redon, confrontés à la création d'un prieuré de l'abbaye de Marmoutier, au début du XII ${ }^{\mathrm{e}} \mathrm{s}$. et que par ailleurs aucune opération archéologique d'envergure n'a été menée au sein de l'agglomération.

Le propos est allègrement servi par les photographies d'Hervé Ronné et par une large palette iconographique où l'on peut distinguer plusieurs chartes issues des Archives départementales du Morbihan relatives aux prieurés fondés à proximité du château aux $\mathrm{XI}^{\mathrm{e}}$ et $\mathrm{XII}^{\mathrm{e}} \mathrm{s}$. On n'échappe pas à une iconographie par ailleurs bien connue illustrant les travaux des champs (p. 26 et 116), l'adoubement (p. 69), les activités commerciales (p. 108 et 110), une motte de la tapisserie de Bayeux (p. 17) et la guerre de Succession de Bretagne (p. 44, 45 et 53). Plus intimes sont les représentations des seigneurs du lieu comme Jean II de Rohan selon un dessin de Gaignières (p. 115), sur une des cheminées du château au sein de sa devise «A plus » (p. 115), figuré sur l'une des peintures de la chapelle du Stival près de Pontivy (p. 125) ou encore sur un vitrail préservé près de Morlaix (p. 89). On n'échappe pas au tombeau de marbre noir et blanc d'Olivier de Clisson et de son épouse Marguerite de Rohan réalisé au début du Xve $\mathrm{s}$. par un atelier de Tournai. Préservé lors de la Révolution, il est présenté dans l'église Notre-Dame-du-Roncier, proche du château. De ce dernier on retient avant tout la façade gothique flamboyante du logis érigée vers 1500 par le grand constructeur de châteaux que fut Jean II de Rohan et pourvue d'une riche décoration présentée par Diégo Mens, conservateur du patrimoine. La fin de l'ouvrage invite à une déambulation dans les rues de la petite cité médiévale aux maisons à pans de bois préservées et valorisées par des couleurs chatoyantes, nonobstant la disparition de nombreuses autres, des halles et de la majeure partie des murailles et de toutes les portes de la ville médiévale. 
Le travail monographique souffre de certains travers quand l'a. décrit Josselin comme une des " plus belles villes du duché » (p. 77) ou de ville " bénie des dieux » à l'époque médiévale (p. 95). Nous sommes bien loin de cités comme Rennes, Nantes ou Dinan, ne serait-ce qu'au regard de la superficie enclose ou de l'absence d'un couvent mendiant à Josselin. L'exploitation du travail inégalé de JeanPierre Leguay (Jean-Pierre LeguaY, Un réseau urbain au Moyen Agge : les villes du duché de Bretagne aux $X^{e}{ }^{e} t_{X I V^{e}}$ siècles, Paris, Maloine, 1981) aurait permis une meilleure immersion dans l'univers de la soixantaine de villes médiévales bretonnes que cet historien qualifie parfois de simples « bourgades ». A contrario, on peut employer le vocable de « capitale » pour Josselin, même si cette dernière a correspondu à un fief à géométrie variable, entre l'apogée de la vicomté de Porhoët au XII ${ }^{\mathrm{e}}$ s., son partage en 1249 , son acquisition par Olivier de Clisson en 1370 et l'union de cette seigneurie avec la vicomté de Rohan en 1407. L'a. souligne l'absence des seigneurs de Josselin de 1249 à 1370, correspondant selon l'a. à un véritable « déclin » ce qui mérite certainement d'être nuancé, puis l'importance de certains personnages qui réinvestissent et rebâtissent le château et des monuments urbains, comme Olivier de Clisson et Jean II de Rohan. L'a. semble méconnaître le réseau castral tissé autour de ce chef-lieu, avec d'autres châteaux comme Blain, Rohan, La Chèze ou Pontivy, qui eurent quelquefois la préférence des uns puis des autres, $\mathrm{du}$ $\mathrm{XIV}^{\mathrm{e}}$ au XVI ${ }^{\mathrm{e}}$ s., sans même compter des manoirs et autres résidences de plaisance. On peut aussi s'étonner de l'absence de certaines illustrations comme l'atlas cadastral de 1832 présentant une ville au tracé encore médiéval ceinte de faubourgs ou encore le plan cavalier de Damien Cabiron et Anne Holmberg réalisé il y a peu sous l'égide des petites cités de caractère de Bretagne et employé sur les panneaux de signalétique patrimoniale de Josselin. L'histoire des propriétaires successifs de la ville qui en ont fait les « grandes heures » et l'évocation de faits guerriers comme le combat des Trente nous privent d'une vision un peu plus topographique et intimiste de la ville et de certains quartiers comme Sainte-Croix, faubourg implanté au-delà de l'Oust dont la canalisation a fait disparaître l'ancien pont et les pittoresques moulins qui complétaient le paysage médiéval.

On aurait également aimé disposer d'une introduction présentant les objectifs de l'a. et d'une conclusion élargissant le propos aux cités voisines du centre Bretagne et incitant d'autres personnes à suivre l'exemple de cette publication qui peut se lire de bout en bout mais aussi être feuilletée. Trop peu de villes castrales bretonnes disposent ainsi d'un bel ouvrage retraçant leur histoire médiévale, en associant un texte issu de recherches récentes et une riche iconographie, si ce n'est par exemple Châteaubriant (Christian Bouvet, Châteaubriant au Moyen Âge : des origines à la fin $d u X V^{e}$ siècle, Châteaubriant, Histoire et patrimoine du pays de Châteaubriant, 2015). Ces initiatives permettent enfin de renouveler des monographies parfois vieilles de plus d'un siècle et encore réimprimées, en dépit des progrès de la recherche historique et des congrès qui ont notamment pu y être organisés par la Société d'histoire et d'archéologie de Bretagne depuis plusieurs décennies.

Patrick KeRNÉVEZ

CRBC - Université de Brest Bretagne Occidentale 